\title{
PAL SPME Arrow-evaluation of a novel solid-phase microextraction device for freely dissolved PAHs in water
}

\author{
Andreas Kremser ${ }^{1}$ • Maik A. Jochmann ${ }^{1}$ - Torsten C. Schmidt ${ }^{1}$
}

Received: 6 October 2015 / Revised: 5 November 2015 / Accepted: 9 November 2015 /Published online: 16 December 2015

(C) The Author(s) 2015. This article is published with open access at Springerlink.com

\begin{abstract}
After more than 25 years, solid-phase microextraction (SPME) has gained widespread acceptance as a wellautomatable and flexible microextraction technique, while its instrumental basis remained mostly unchanged. The novel PAL (Prep And Load solution) SPME Arrow combines the advantages of SPME with the benefits of extraction techniques providing larger sorption phase volumes such as stir bar sorptive extraction (SBSE). It thereby avoids the inherent drawbacks of both techniques such as limitations in method automation in the case of SBSE, as well as the small sorption phase volumes and the lacking fiber robustness of classical SPME fibers. This new design is based on a robust stainless steel backbone, carrying, the screw connection to the PAL sampler, the enlarged sorption phase, and an arrow-shaped tip for conservative penetration of septa (hence the name). An outer capillary encloses this phase apart from enrichment and desorption processes and rests against the tip during transfer and penetrations, resulting in a homogeneously closed device. Here, we present an evaluation and a comparison of the novel PAL SPME Arrow with classical SPME fibers, extracting polycyclic aromatic hydrocarbons (PAHs) as model analytes, from the freely dissolved fraction in lab water and groundwater via direct immersion using polydimethylsiloxane (PDMS) as common sorption phase material. Limits of detection, repeatabilities, and extraction yields were determined for the PAL SPME Arrow and compared to data of
\end{abstract}

Electronic supplementary material The online version of this article (doi:10.1007/s00216-015-9187-z) contains supplementary material, which is available to authorized users.

Torsten C. Schmidt

torsten.schmidt@uni-due.de

1 Instrumental Analytical Chemistry, University of Duisburg-Essen, Universitätsstraße 5, 45141 Essen, Germany classical SPME fibers and SBSE bars. Results indicate a significant benefit in extraction efficiency due to the larger sorption phase volume. It is accompanied by faultless mechanical robustness and thus better reliability, especially in case of prolonged, unattended, and automated operation. As an exemplary application, the water-soluble fraction of PAHs and derivatives in a roofing felt sample was quantified.

Keywords PAL SPME Arrow · SPME · Microextraction · $\mathrm{PDMS} \cdot \mathrm{PAH}$

\section{Introduction}

Solid-phase microextraction (SPME) was developed by Belardi and Pawliszyn in 1989 [1] and is nowadays the most popular and most frequently used microextraction technique [2]. The reasons for this popularity are its operational simplicity, short extraction times, possibility of a fully automated operation, avoidance of organic solvents [3], as well as its direct and straightforward thermodesorption into a gas chromatographic system. Furthermore, SPME combines matrix separation of analytes with a concentrating step [4] and can be used for in situ, in-field, and even in vivo sampling $[2,5,6]$. However, apart from many advantages, it also has drawbacks, including the limited mechanical robustness of the fiber [7-10] and the rather small sorption phase volume of the commercially available fibers $[2,8,11]$.

In order to overcome especially the latter disadvantage, the SPME-related technique stir bar sorptive extraction (SBSE) was developed. SBSE provides a significantly larger extraction phase in the order of $100 \mu \mathrm{L}$ compared to about $1 \mu \mathrm{L}$ with classical SPME, but loses the advantage of full automation, as the SBSE bar has to be recovered from the sample, dried, and introduced into a special thermodesorption unit in a manual process. 
Recently, a novel SPME-related extraction device named PAL (Prep And Load solution) SPME Arrow was developed. As the first alternative in this field to be based on a completely redesigned, automatable fiber, it aims at combining the advantages of the classical SPME fiber and the SBSE, while remediating the main inherent disadvantages of these techniques. It is presented in Fig. 1 alongside a classical SPME fiber, and its properties will be thoroughly discussed in the "Results and discussion" section.

Polycyclic aromatic hydrocarbons (PAHs) are abundant environmental contaminants originating from both anthropogenic as well as natural sources, which typically involve incomplete combustion processes such as forest fires or burning of fossil fuels $[5,12]$. PAHs are also contained in bitumenrelated products that are used in various fields of construction, especially due to their hydrophobic properties, which make them a widespread choice for waterproofing applications [13]. While the fumes and vapors that originate from production and handling of such materials are already suspected to represent occupational risks in terms of exposure to PAHs [13], the leaching of the latter compounds into runoff water was mostly neglected in the past, often due to insufficient detection limits of the analytical methods [14].

Typical SPME LODs and LOQs for measurements of PAH in water are in the nanogram-per-liter range, depending on utilized sorption phase and analytical conditions [15]. In this context of increasing analytical demands, PAHs were used as representative and well comparable analytes in order to determine to which extent PAL SPME Arrow surpasses limitations of classical SPME fibers without compromising original SPME advantages.

Polydimethylsiloxane (PDMS) was used as common sorption phase material [16] because, just like the aforementioned analytes, it enables effective comparison of results with literature.

\section{Experimental section}

Reagents and materials Optimization and calibration were carried out by using a PAH standard (SV Calibration Mix \#5/
610 PAH) purchased from Restek (Bellefonte, PA). The standard contains 16 PAHs in methylene chloride at a concentration of $2 \mathrm{~g} \mathrm{~L}^{-1}$, respectively (see Table $\mathrm{S} 1$ in the electronic supplementary material (ESM)). Analytical-grade methanol (KMF Laborchemie, Lohmar, Germany) and lab water from a PURELAB Ultra analytic water purification system (ELGA LabWater, Celle, Germany) were used as solvents for stock, standard, and sample preparation. In the case of groundwater samples, the water was kindly supplied by LINEG (Kamp-Lintfort, Germany) and filtered through medium-dense MN 615 cellulose filters with a thickness of $0.16 \mathrm{~mm}$ and a surface weight of $70 \mathrm{~g} \mathrm{~m}^{-2}$, which were obtained from Macherey-Nagel (Düren, Germany). G200 DD sanded roofing felt according to EN 13969 and EN 14967 was purchased at a Hornbach building supply store (Essen, Germany).

Standard solutions From the PAH calibration mix, a methanolic stock solution with a concentration of $1 \mathrm{mg} \mathrm{L}^{-1}$ was prepared and stored in a $20-\mathrm{mL}$ amber screw-cap headspace vial, with silicone/PTFE septa and no headspace (BGB Analytik, Boeckten, Switzerland), in the refrigerator at $4{ }^{\circ} \mathrm{C}$. From this stock solution, aqueous standard dilutions were prepared and stored in the same manner. Hamilton glass syringes (Hamilton, Bonaduz, Switzerland) and Blaubrand ${ }^{\circledR}$ bulb pipettes (Brand, Wertheim, Germany) were used for stock, dilution standard, and sample preparation.

The PDMS tubes which were used as extraction phases for PAL SPME Arrows were also obtained from BGB Analytik.

Roofing felt samples were prepared by cutting the material into pieces of $2 \mathrm{~mm} \times 4 \mathrm{~cm}$ (approx. $300 \mathrm{mg}$ ) and adding one of these pieces to vials containing $19 \mathrm{~mL}$ of lab water. Pieces were deliberately used as a whole since further disintegration would have resulted in a larger total surface area of the material and therefore an overestimation of PAH leaching into water.

Since PAHs readily adsorb to almost any available surface and are thereby lost to solid-phase extraction processes, it is reasonable to calculate their equilibrium ratios that are adsorbed to the surfaces available in the prepared samples in order to avoid biased results [17].

\section{Classical SPME fiber}

Fig. 1 Sketch of a classical SPME Arrow. The SPME fiber possesses a $100-\mu \mathrm{m} \times 10-\mathrm{mm}$, $0.6-\mu \mathrm{L}$ sorption phase. The PAL SPME Arrow is equipped with a $250-\mu \mathrm{m} \times 30-\mathrm{mm}, 15.3-\mu \mathrm{L}$ sorption phase, respectively, has a stainless steel inner core with a diameter of $0.4 \mathrm{~mm}$, and an overall external diameter of $1.5 \mathrm{~mm}$

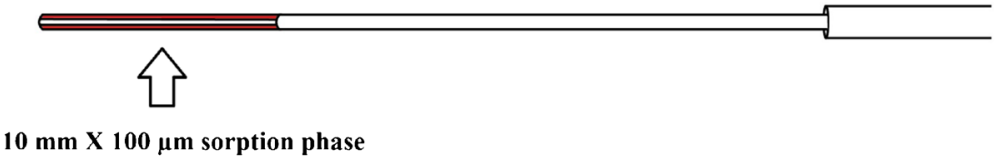

PAL SPME Arrow

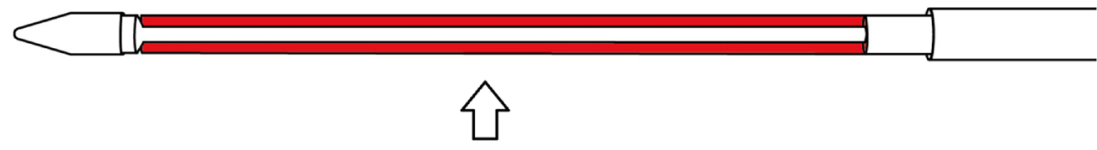

$30 \mathrm{~mm} \times 250 \mu \mathrm{m}$ sorption phase 
Partitioning of analytes into the headspace was calculated [18] as, e.g., $0.16 \%$ for naphthalene, which is the most volatile PAH. Analyte loss due to sorption to glassware was calculated as well [19], with adsorbed analyte fractions of, e.g., $0.3 \%$ in case of pyrene. Sorption of analytes to the PTFE septa of sample vial caps was the strongest influence in this context, with an equilibrium value of $3.8 \%$ for pyrene [17].

Therefore, $3.8 \%$ can be considered to be the maximum value here, resulting in a total loss of adsorbed analytes below $5 \%$, which was neglected during the further course of this study. In order to ensure proper sample equilibration prior to measurement series, samples were incubated at room temperature for at least $24 \mathrm{~h}$ prior to extraction.

Samples were stirred with self-constructed stir bars prepared from $1.5 \times 10-\mathrm{mm}$ iron cylinder bolts enclosed in fused silica.

GC/MS instrumentation and parameters All analyses were carried out on a Shimadzu GCMS-QP2010 Ultra (Shimadzu Deutschland GmbH, Duisburg, Germany). Thermal desorption of the extracted analytes was carried out using a split/splitless injector, which was set to a temperature of $280{ }^{\circ} \mathrm{C}$. The injector was equipped with a Restek ( $2 \mathrm{~mm}$ i.d. $\times 5 \mathrm{~mm}$ o.d. $\times 95 \mathrm{~mm}$ length) splitless liner (BGB Analytik, Boeckten, Switzerland). The thermal desorption time was $5 \mathrm{~min}$, and after a splitless time of $6 \mathrm{~min}$, the split ratio was set to $10: 1$.

Analyte separation was accomplished on a $30-\mathrm{m} \times 0.25$ $\mathrm{mm}$ Rxi ${ }^{\circledR}$-PAH column (Restek, Bellefonte, PA) with a $0.1-\mu \mathrm{m}$ film thickness. As carrier gas, helium 5.0 (Air Liquide, Oberhausen, Germany) with a flow of $1.5 \mathrm{~mL} \mathrm{~min}^{-1}$ was used. The GC temperature program started with a 5 -min standby at $40^{\circ} \mathrm{C}$, followed by a first temperature ramp of $50{ }^{\circ} \mathrm{C} \mathrm{min}^{-1}$ up to $110{ }^{\circ} \mathrm{C}$, a second ramp of $5{ }^{\circ} \mathrm{C} \min ^{-1}$ to $240{ }^{\circ} \mathrm{C}$, and a third ramp of $50{ }^{\circ} \mathrm{C} \mathrm{min}^{-1}$ to a final temperature of $320^{\circ} \mathrm{C}$, which was maintained for $5 \mathrm{~min}$ for cleanup purposes. The transfer line and ion source were both set to $250{ }^{\circ} \mathrm{C}$, respectively. Retention times varied between 8.70 and $49.48 \mathrm{~min}$ for naphthalene- $\mathrm{d}_{8}$ and benzo(ghi)perylene (see Table S2 in ESM), respectively.

In accordance with literature [20], the chosen chromatographic conditions enabled sufficient separation of all target compounds. A resulting chromatogram is included in the ESM (Fig. S4), as well as detailed mass spectrometric detection parameters.

Extraction procedure Samples were extracted by a PAL RTC autosampler, which was equipped with SPME fibers $(100 \mu \mathrm{m} \times 10 \mathrm{~mm}, 0.6 \mu \mathrm{L})$ and PAL SPME Arrows $(250 \mu \mathrm{m} \times 20 \mathrm{~mm}, 10.2 \mu \mathrm{L}$ ) (all from CTC Analytics AG, Zwingen, Switzerland). The 20-mm-long sorption phase was chosen for PAL SPME Arrow to facilitate constant and complete submersion during extraction.
Due to the larger diameter of PAL SPME Arrow in contrast to traditional SPME fibers, the openings of the PAL tool needle guide, the GC injector, and the SPME fiber conditioning station had to be widened to approximately $1.8 \mathrm{~mm}$.

Samples were stored in their tray at room temperature $\left(23^{\circ} \mathrm{C}\right)$. Prior to extraction, they were transferred to a selfconstructed stirring station based on an IKA-Mag RCT basic (IKA-Werke GmbH \& CO KG, Staufen, Germany). In this station, samples were continuously stirred at 1500 rounds per minute (rpm) and $35{ }^{\circ} \mathrm{C}$, first for a temperature preequilibration time of $10 \mathrm{~min}$ and afterwards during sample extraction. Simultaneous to the first $5 \mathrm{~min}$ of sample preequilibration time, the SPME fiber or PAL SPME Arrow was preconditioned in the SPME fiber conditioning station at $200{ }^{\circ} \mathrm{C}$ under a stream of nitrogen 5.0.

After the sample pre-equilibration time, the sample vials' septa were pierced by the fiber and the sorption phase was immersed into the continuously stirred sample for $70 \mathrm{~min}$. The sample vial penetration depth was thereby set to $55 \mathrm{~mm}$, in order to ensure constant and complete immersion of the sorption phase.

Once extraction was completed, the fiber was transferred into the $\mathrm{GC}$ injector for thermal desorption at $280{ }^{\circ} \mathrm{C}$. Subsequently, it was cleaned for $15 \mathrm{~min}$ in the SPME fiber conditioning station at $200{ }^{\circ} \mathrm{C}$. The PAL RTC sequence was interlocked so that the subsequent equilibration and extraction were carried out during the GC run of the previous sample in order to reduce overall analysis time.

\section{Results and discussion}

Fiber properties PAL SPME Arrow is based on a stabilizing stainless steel inner rod that runs continuously through the entire fiber, carrying the cylindrically shaped sorption phase and connecting the upper parts of the device to its solid tip, which is shown in Fig. S2 of the ESM, and specially designed to allow gentle penetration of injector and sample vial septa. This tip also retains the sorption phase, which is attached to the inner rod, and furthermore enables PAL SPME Arrow's capability to enclose this sorption phase during transfer processes. This is an important difference to the traditional SPME fiber, which only allows for the retraction of the latter, with its outer capillary more open to external, potentially adverse influences such as contaminations from ambient air. Furthermore, an open capillary faces significant resistance during penetration processes, in contrast to a PAL SPME Arrow in its closed state. Its outer capillary rests against the solid tip, resulting in a homogeneously closed fiber since both parts possess the same diameter.

A sketch of a conventional SPME fiber and a PAL SPME Arrow is shown in Fig. 1. Pictures comparing the points and 
sorption phases of both instruments are included in the ESM (Figs. S1 and S2).

Classical SPME fibers can cause coring of injector septa due to their open tubular tip [2]. Based on own experiences, exchange of the septa of gas chromatographic systems, which are subject to regular SPME measurements, is required after approximately 100 injections to avoid leakages and introduction of septum material into the liner.

Using PAL SPME Arrow, the wear of injector septa diminished due to the specially designed tip. Despite the enlarged diameter compared to the classical fiber, at least 200 injections without coring, abrasion, or leakage are possible.

PAL SPME Arrow demonstrated faultless mechanical reliability over the entire course of these studies. In our experience, classical SPME fibers are more fragile, typically requiring replacement after 100 to 200 injections due to bending of the fibers (an exemplary picture is included in the ESM). These values seem to be typical and are also encountered in literature [7, 9, 10]. Active agitation of the sample vial (instead of the liquid sample via stirring) by the standard PAL agitator may decrease this value even further since the fiber material is weakened by being constantly bent into alternating directions.

The main reason for this change in mechanical reliability is the increased diameter of the fibers' outer capillary, which is $1.5 \mathrm{~mm}$ in contrast to approx. $0.7 \mathrm{~mm}$ in the case of the classical gauge 23 SPME fiber. In addition, the tip of PAL SPME Arrow not only conserves septa during penetration but thereby also lowers the resistance, which has to be overcome.

Extraction optimization In general, PAL SPME Arrow and classical SPME fibers require the same optimization procedure. For the here applied direct immersion (DI) extraction, the important optimization steps are evaluation of extraction time and stirring velocity [6].

In Fig. 2, the influence of stirring rate and extraction time is shown exemplarily for four of the 16 EPA PAHs, with achieved results confirming expectations according to literature $[2,15,21]$.

For the optimized PAL SPME Arrow method, an extraction time of 70 min was chosen. Apparently, this technique represents a reasonable compromise in this context. Classical SPME fibers typically require approx. $30 \mathrm{~min}$ [15] of extraction time in order to reach an equilibrium state, and alternative extraction techniques with larger sorption phases such as SBSE may require timeframes of up to $14 \mathrm{~h} \mathrm{[22].}$

In Fig. $2 b$, the influence of the stirring rate between 0 and 1500 rounds per minute (rpm) is shown. In accordance with the SPME extraction theory [2], an increased stirring rate leads to a higher mass transfer in the system, since the diffusion layer around the fiber coating is minimized and
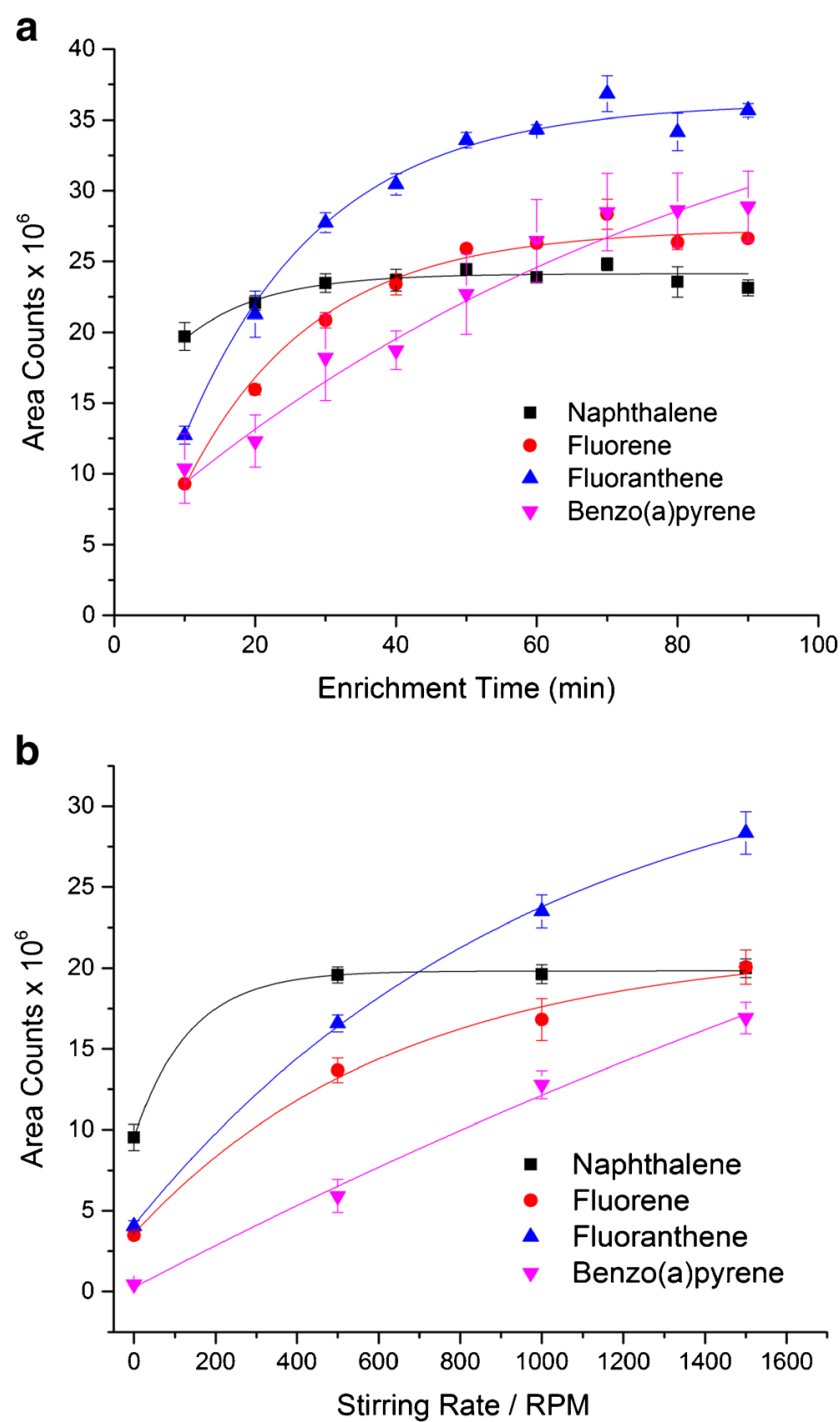

Fig. 2 Extraction optimization measurements for PAL SPME Arrow extractions of PAH from aqueous solutions: a extraction time (stirring rate kept at $1500 \mathrm{rpm}$ ), b stirring rate (extraction time kept at $70 \mathrm{~min}$ ). All samples contained $500 \mathrm{ng} \mathrm{L}^{-1}$ of PAHs and were extracted by a PAL SPME Arrow with a PDMS sorption phase $(250 \mu \mathrm{m} \times 20 \mathrm{~mm}, 10.2 \mu \mathrm{L})$. Exponential trend lines were added via Origin Pro 2015

thus the equilibrium is attained faster. For the optimized method, the maximum possible stirring rate of $1500 \mathrm{rpm}$ was used.

Since the typical behavior of decreasing extraction yields at higher temperatures caused by smaller partition coefficients of the analytes between the extraction phase and the sample matrix [2] could be observed in our preliminary measurements as well, the lowest possible temperature of $35^{\circ} \mathrm{C}$ was used for all sample extractions. See Fig. S5 in the ESM for the corresponding optimization results.

Extraction efficiency To determine the effects of the enlarged sorption phases in the case of PAL SPME Arrow, a comparison with classical SPME fibers was performed. Prior to 
sample measurements, theoretically extracted analyte amounts were calculated with Eq. (1) [21]:

$m_{f}=\frac{K_{f s} V_{f} V_{s} c_{0}}{K_{f s} V_{f}+V_{s}}$

where $m_{f}$ is the extracted mass of analyte in the polymeric sorption phase under equilibrium conditions and $V_{f}$ and $V_{s}$ are the volumes of the polymer and the aqueous sample, respectively. The initial amount of each analyte present in the aqueous samples with a volume of $19 \mathrm{~mL}$ and an initial analyte concentration $\left(c_{0}\right)$ of $10 \mathrm{ng} \mathrm{L}^{-1}$ was $190 \mathrm{pg}$.

The distribution constants $K_{f s}$ for the analytes' phase transition from the aqueous solution into the PDMS sorption phase were calculated from literature parameters [23] and Eq. (2), yielding the results included in Table 1. The letters $E, S, A, B$, and $V$ thereby denote the solute descriptors according to the Abraham model for excess molar refraction, dipolarity/polarizability, overall hydrogen bond acidity, overall hydrogen bond basicity, and McGowan volume, respectively [24].

$$
\begin{aligned}
\log K_{f s}= & 0.246+0.568 E-1.305 S-2.565 A-3.928 B \\
& +3.573 V
\end{aligned}
$$

According to Table 1, PAL SPME Arrows can be expected to exhibit improved extraction yields when compared to classical SPME fibers with a ratio of up to 12.2 for PAHs. In the case of the SBSE bars, the further improvement in relation to PAL SPME Arrow has a ratio of up to 2.2. Especially for molecularly larger compounds with a $\log K_{f s}$ of approx. 5 or larger, differences in extraction efficiency between PAL SPME Arrow and SBSE are negligible. Obviously, the effect of a further increase in sorption phase dimensions peaks in the range where PAL SPME Arrow is situated. The critical relation here is the phase ratio between sample and sorption phase. While these results were calculated for 20-mL vials, the SBSE technique is probably better suited for analysis of larger sample volumes, which are however less straightforward to automate.

Further investigation on the extraction behavior of PAL SPME Arrow was conducted by calculating the recoveries that are to be expected theoretically from PDMS-based extraction techniques with different phase volumes. We selected a commonly available variant of classical SPME fibers, a PAL SPME Arrow, and an SBSE device as representative examples. Using $K_{f s}$ values from literature [23], we calculated the theoretically extracted percentages for the aforementioned extraction phases and three model analytes under equilibrium conditions (Fig. 3).

In order to evaluate these calculated values, the depletion SPME method [25] was used to determine the extracted percentages of analytes out of a sample with an initial concentration of $50 \mathrm{ng} \mathrm{L}^{-1}$ for a single extraction. The latter was carried out either by a classical SPME fiber $(100 \mu \mathrm{m} \times 10 \mathrm{~mm}$, $0.6 \mu \mathrm{L})$ or a PAL SPME Arrow $(250 \mu \mathrm{m} \times 20 \mathrm{~mm}$, $10.2 \mu \mathrm{L}$ ). This method is based on performing depletion extractions by extracting and measuring samples multiple times. The declining, logarithmical peak areas are then plotted against the number of consecutive extractions, yielding a linear regression, whose slope $b$ then enables calculation of the extraction ratio $E$ from $\log (1-E)$ [25].

The results of these measurements can be seen in Table 2 and are in good agreement with literature [8], as well as the previously calculated values in Table 1 . This is also visible when plotting calculated against measured results with a linear

Table 1 Calculated $\log K_{f s}$ and $m_{f}$ values for ten exemplary PAHs included in this work, determined for a SPME fiber $(100 \mu \mathrm{m} \times 10 \mathrm{~mm}$, $0.6 \mu \mathrm{L})$, a PAL SPME Arrow $(250 \mu \mathrm{m} \times 20 \mathrm{~mm}, 10.2 \mu \mathrm{L})$, and an SBSE

bar $(500 \mu \mathrm{m} \times 20 \mathrm{~mm}, 47 \mu \mathrm{L})$ for a $c_{0}$ of $10 \mathrm{ng} \mathrm{L}^{-1}$, sorted by ascending $\log K_{f s}$ value, based on solute descriptors from literature [23]

\begin{tabular}{lccccc}
\hline Compound & $\log K_{f s}$ & $\begin{array}{l}m_{f}(\text { SPME fiber }) \\
(\mathrm{pg})\end{array}$ & $\begin{array}{l}m_{f}(\text { PAL SPME Arrow }) \\
(\mathrm{pg})\end{array}$ & $\begin{array}{l}m_{f} \text { (SBSE bar) } \\
(\mathrm{pg})\end{array}$ & $\begin{array}{l}\text { Ratio of extracted masses } \\
\text { PAL SPME Arrow vs. SPME } \\
\text { fiber/SBSE bar vs. PAL SPME Arrow }\end{array}$ \\
\hline Naphthalene & 2.8991 & 4.6 & 56.7 & 125.8 & $12.2 / 2.2$ \\
Acenaphthene & 3.4196 & 14.6 & 111.2 & 164.7 & $7.6 / 1.5$ \\
Fluorene & 3.6313 & 22.6 & 132.4 & 173.6 & $5.9 / 1.3$ \\
Anthracene & 3.8933 & 37.6 & 153.5 & 180.7 & $4.1 / 1.2$ \\
Fluoranthene & 4.2939 & 72.8 & 173.6 & 186.2 & $2.4 / 1.1$ \\
1,2-Benzanthracene & 4.9443 & 139.7 & 186.1 & 189.1 & $1.3 / 1.0$ \\
Benzo(a)pyrene & 4.9744 & 142.2 & 186.3 & 189.2 & $1.3 / 1.0$ \\
Benzo(b)fluoroanthene & 5.0941 & 151.4 & 189.2 & 189.4 & $1.2 / 1.0$ \\
Benzo(ghi)perylene & 5.6407 & 177.2 & 187.9 & 189.8 & $1.1 / 1.0$ \\
Dibenz(a,h)anthracene & 5.9609 & 183.6 & 189.6 & 189.9 & $1.0 / 1.0$
\end{tabular}


Fig. 3 Theoretically extracted percentages for three extraction techniques and exemplary PAHs under equilibrium conditions calculated for an aqueous sample volume of $19 \mathrm{~mL}$ with indicated PDMS volumes and $\log K_{f}$ values from literature [23]

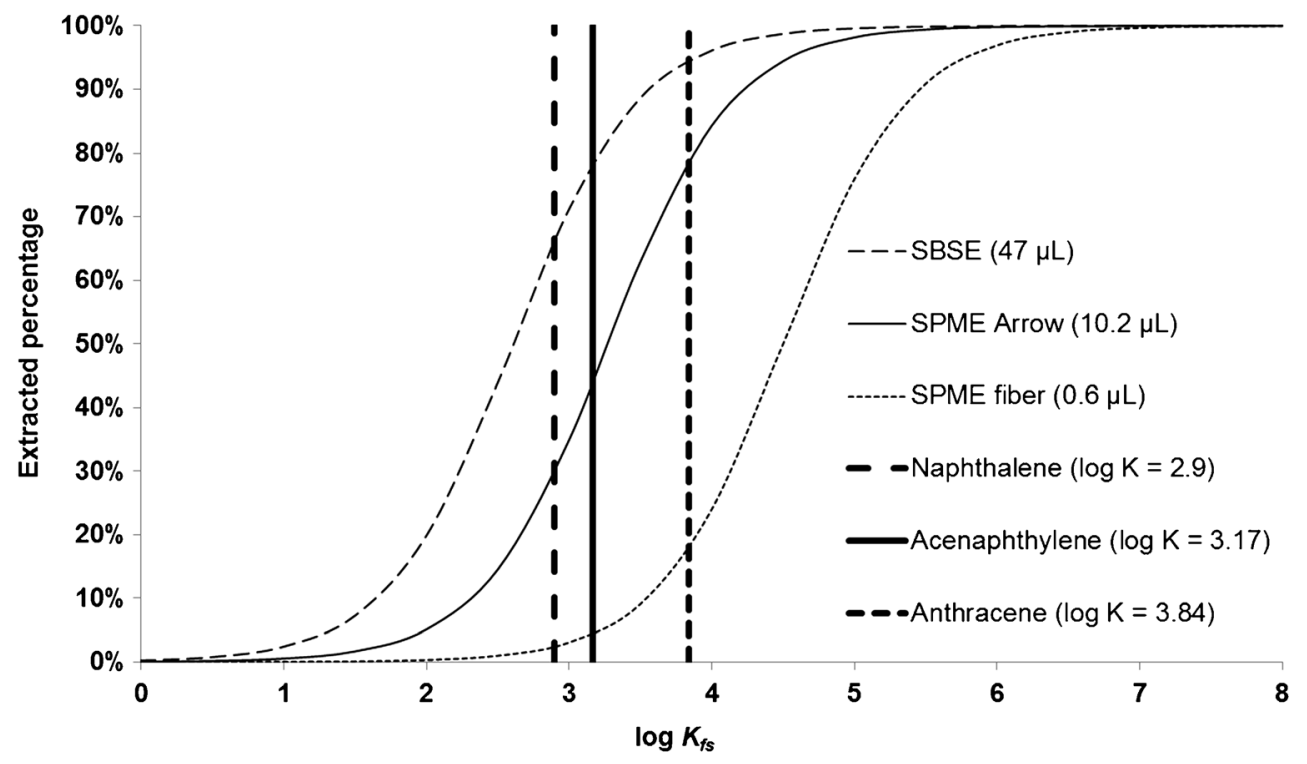

trend line. An example for such plots can be found in Fig. S7 in the ESM, exhibiting a correlation coefficient of 0.9777 . Depletion curves of these measurements and their corresponding linear correlations and trend lines are shown in Figs. 4 and S6. The slope of the logarithmic depletion curves and their linear correlations are also included in Table 2, demonstrating sufficiently good correlations $(>0.98)$ for all analytes. These measurements were also carried out for the largest available PAL SPME Arrow sorption phase variant $(250 \mu \mathrm{m} \times 30 \mathrm{~mm}, 15.3 \mu \mathrm{L})$, and the results are included in the ESM (Table S2).
The increased extraction yields of PAL SPME Arrow are also visible in Fig. 4 by comparing the depletion curves from these experiments that were either generated using a classical SPME fiber (a) or PAL SPME Arrow (b), since the latter showed a more rapid depletion of analytes in the samples.

Comparison to literature To enable a statistical comparison of achievable detection limits for PAL SPME Arrow with classical SPME fibers, we determined the method detection limits (MDL) according to Keith et al. [26], as well as relative standard deviations.
Table 2 Slopes, correlation coefficients, and extracted percentages of the performed depletion experiments according to Zimmermann et al. [25] for samples containing $19 \mathrm{~mL}$ of water and an initial concentration of $50 \mathrm{ng} \mathrm{L}^{-1}$ PAHs for the first extraction by a classic SPME fiber $(100 \mu \mathrm{m} \times 10 \mathrm{~mm}, 0.6 \mu \mathrm{L})$ and a PAL SPME Arrow $(250 \mu \mathrm{m} \times 20 \mathrm{~mm}, 10.2 \mu \mathrm{L})$

\begin{tabular}{|c|c|c|c|c|c|c|}
\hline \multirow[t]{2}{*}{ Compound } & \multicolumn{3}{|c|}{ SPME fiber } & \multicolumn{3}{|c|}{ PAL SPME Arrow } \\
\hline & Slope & $R^{2}$ & $E(\%)$ & Slope & $R^{2}$ & $E(\%)$ \\
\hline Naphthalene & -0.023 & 0.9903 & 5.2 & -0.084 & 0.9915 & 17.5 \\
\hline Acenaphthylene & -0.028 & 0.9842 & 6.2 & -0.134 & 0.9980 & 26.6 \\
\hline Acenaphthene & -0.041 & 0.9946 & 9.0 & -0.144 & 0.9907 & 28.2 \\
\hline Fluorene & -0.050 & 0.9927 & 10.9 & -0.146 & 0.9902 & 28.6 \\
\hline Phenanthrene & -0.060 & 0.9972 & 12.9 & -0.163 & 0.9959 & 31.4 \\
\hline Anthracene & -0.071 & 0.9930 & 15.1 & -0.225 & 0.9943 & 40.5 \\
\hline Pyrene & -0.097 & 0.9956 & 20.1 & -0.254 & 0.9993 & 44.3 \\
\hline Fluoroanthene & -0.096 & 0.9972 & 19.9 & -0.239 & 0.9996 & 42.3 \\
\hline 1,2-Benzanthracene & -0.137 & 0.9925 & 27.1 & -0.307 & 0.9946 & 50.7 \\
\hline Chrysene & -0.071 & 0.9235 & 15.1 & -0.278 & 0.9871 & 43.4 \\
\hline Benzo(b)fluoroanthene & -0.172 & 0.9938 & 32.7 & -0.317 & 0.9964 & 51.8 \\
\hline Benzo(k)fluoroanthene & -0.176 & 0.9878 & 33.4 & -0.412 & 0.9937 & 61.3 \\
\hline Benzo(a)pyrene & -0.156 & 0.9958 & 30.2 & -0.330 & 0.9854 & 53.2 \\
\hline Indeno( $1,2,3$ cd)pyrene & -0.159 & 0.9845 & 30.7 & -0.367 & 0.9897 & 57.0 \\
\hline Dibenz(ah)anthracene & -0.142 & 0.9926 & 27.8 & -0.465 & 0.9994 & 65.7 \\
\hline Benzo(ghi)perylene & -0.140 & 0.9967 & 27.6 & -0.418 & 0.9999 & 61.9 \\
\hline
\end{tabular}


Fig. 4 Depletion curves for three exemplary PAHs, extracted by a classical SPME fiber $(100 \mu \mathrm{m} \times$ $10 \mathrm{~mm}, 0.6 \mu \mathrm{L})(\mathbf{a})$ and a PAL SPME Arrow $(250 \mu \mathrm{m} \times 20 \mathrm{~mm}$, $10.2 \mu \mathrm{L})(\mathbf{b})$ a

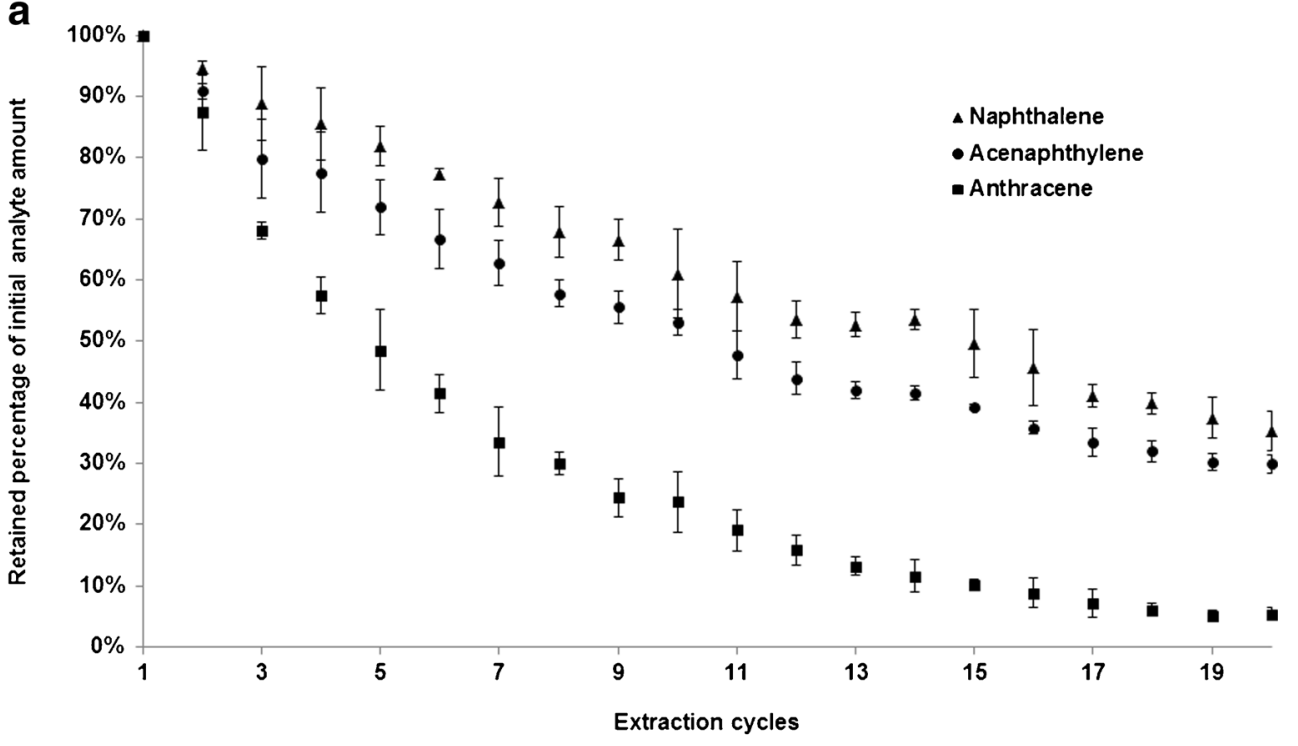

b

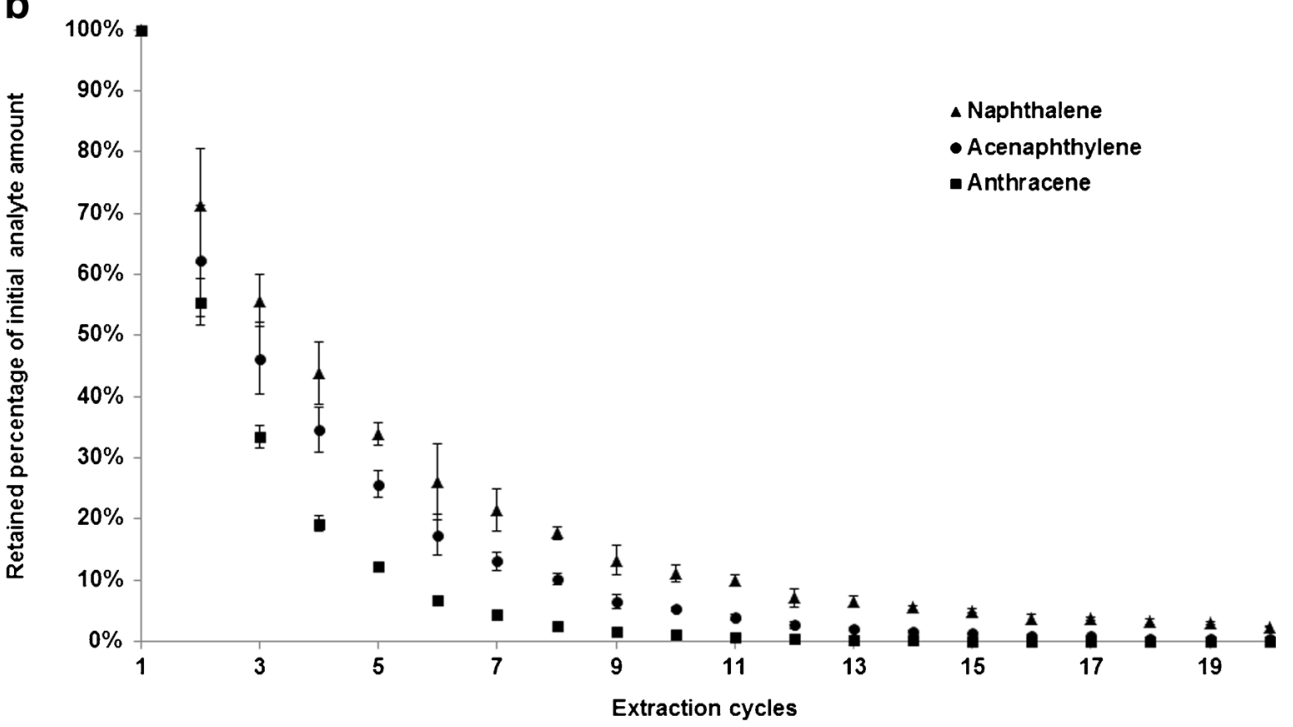

Using PAL SPME Arrow $(250 \mu \mathrm{m} \times 20 \mathrm{~mm}, 10.2 \mu \mathrm{L})$, it was possible to calibrate in concentration ranges as low as 0.5 to $2.5 \mathrm{ng} \mathrm{L}^{-1}$ for all $16 \mathrm{EPA}$ PAHs. Results are displayed in Table 3 in terms of LOD and RSD values for calibrations performed in ultrapure water and filtrated groundwater. Linear ranges and correlation coefficients for these calibrations can be found in the ESM.

In accordance to literature [27], it was impossible to determine freely dissolved PAHs via SPME fiber or PAL SPME Arrow in groundwater samples with a significant content of particulate organic matter (POM).

After removal of POM (along with sorbed compounds) via filtration, spiking of groundwater samples enabled determination of PAHs from the freely dissolved fraction with the following exceptions due to matrix interference: phenanthrene, anthracene, pyrene, fluoroanthene, and indeno(1,2, 3 cd)pyrene, as indicated by the dashes in Table 3 .
Table 4 displays LODs and RSDs for PAL SPME Arrow and comparable techniques. While Cheng et al. [15] extrapolated the LOD values for their classical SPME fibers from the standard deviation of their results at the lowest calibration point $\left(10 \mathrm{ng} \mathrm{L}^{-1}\right)$, the results presented herein were calculated from measurements at $0.5 \mathrm{ng} \mathrm{L}^{-1}$ for reagent water-based samples and at $5 \mathrm{ng} \mathrm{L}^{-1}$ for groundwater samples.

Carrera et al. [22] achieved LODs that are similar to the ones generated with PAL SPME Arrow, by extracting a 100$\mathrm{mL}$ water sample for $14 \mathrm{~h}$ with a $500-\mu \mathrm{m} \times 20$-mm SBSE bar. We calculated the sorption phase volume on these bars to be $47 \mu \mathrm{L}$, which would be approx. threefold larger as the largest available PAL SPME Arrow phase.

Determined MDLs for PAL SPME Arrow are generally more similar to those generated with the SBSE bars and approximately one order of magnitude better than those of the classical SPME fibers. In contrast to SBSE though, these 
Table 3 Calibration results obtained with PAL SPME Arrow $(250 \mu \mathrm{m} \times 20 \mathrm{~mm}, 10.2 \mu \mathrm{L})$ in ultrapure water and groundwater: MDL values (calculated with a $99 \%$ confidence interval) and relative standard deviations (RSD)

\begin{tabular}{|c|c|c|c|c|}
\hline \multirow[t]{2}{*}{ Compound } & \multicolumn{2}{|c|}{ Ultrapure water } & \multicolumn{2}{|c|}{ Groundwater } \\
\hline & $\begin{array}{l}\text { MDL } \\
\left(\text { ng L L }^{-1}\right)\end{array}$ & $\begin{array}{l}\text { RSD (\%) } \\
\text { (at } 10 \mathrm{ng} \mathrm{L}^{-1} \text { ) }\end{array}$ & $\begin{array}{l}\text { MDL } \\
\left(\text { ng L L }^{-1}\right)\end{array}$ & $\begin{array}{l}\text { RSD (\%) } \\
\text { (at } 10 \mathrm{ng} \mathrm{L}^{-1} \text { ) }\end{array}$ \\
\hline Naphthalene & 0.3 & 5.7 & 1.2 & 6.9 \\
\hline Acenaphthylene & 0.2 & 6.0 & 0.9 & 4.8 \\
\hline Acenaphthene & 0.1 & 7.1 & 2.3 & 13.0 \\
\hline Fluorene & 0.2 & 5.6 & 1.9 & 10.6 \\
\hline Phenanthrene & 0.2 & 5.5 & l & / \\
\hline Anthracene & 0.3 & 7.6 & l & / \\
\hline Pyrene & 0.2 & 6.4 & l & l \\
\hline Fluoroanthene & 0.2 & 6.2 & l & / \\
\hline 1,2-Benzanthracene & 0.1 & 6.2 & 0.7 & 3.8 \\
\hline Chrysene & 0.1 & 11.0 & 0.8 & 4.3 \\
\hline Benzo(b)fluoroanthene & 0.2 & 10.5 & 0.6 & 3.4 \\
\hline Benzo(k)fluoroanthene & 0.2 & 8.6 & 0.6 & 3.2 \\
\hline Benzo(a)pyrene & 0.3 & 7.2 & 0.5 & 2.4 \\
\hline Indeno( $1,2,3$ cd)pyrene & 0.8 & 9.2 & l & / \\
\hline Dibenz(ah)anthracene & 0.6 & 11.3 & 0.7 & 3.8 \\
\hline Benzo(ghi)perylene & 0.8 & 11.9 & 0.6 & 3.4 \\
\hline
\end{tabular}

results have been achieved with a fully automated method. The corresponding RSD values are thereby in the range of 5-12\% which is acceptable in such small concentration ranges and in good agreement with literature.
Exemplary leaching experiment For the roofing felt samples, naphthalene and acenaphthylene were the only EPA PAHs that could be measured from the freely dissolved fraction of the sample. This was expected since the material pieces

Table 4 MDL and RSD results obtained with PAL SPME Arrow $(250 \mu \mathrm{m} \times 20 \mathrm{~mm}, 10.2 \mu \mathrm{L})$ for PAHs in water in comparison with literature data for classical SPME fibers and SBSE bars

\begin{tabular}{|c|c|c|c|c|c|c|}
\hline \multirow[t]{2}{*}{ Compound } & \multicolumn{2}{|c|}{ PAL SPME Arrow } & \multicolumn{2}{|c|}{ SPME (Cheng et al.) [15] } & \multicolumn{2}{|c|}{ SBSE (Carrera et al.) [22] } \\
\hline & $\begin{array}{l}\text { MDL } \\
\left(\operatorname{ng~L}^{-1}\right)\end{array}$ & $\begin{array}{l}\text { RSD (\%) } \\
\left(\text { at } 10 \mathrm{ng} \mathrm{L}^{-1} \text { ) }\right.\end{array}$ & $\begin{array}{l}\text { LOD } \\
(\mathrm{SD} X 3)\end{array}$ & $\begin{array}{l}\text { RSD (conc. } \\
\text { at } \mathrm{S} / \mathrm{N}=3 \times 3 \text { ) }\end{array}$ & $\begin{array}{l}\text { LOD (conc. } \\
\text { at } \mathrm{S} / \mathrm{N}=3 \times 3 \text { ) }\end{array}$ & $\begin{array}{l}\operatorname{RSD}(\%) \\
\text { (at } 50 \mathrm{ng} \mathrm{L}^{-1} \text { ) }\end{array}$ \\
\hline Naphthalene & 0.3 & 5.7 & 2.7 & 9.0 & / & l \\
\hline Acenaphthylene & 0.2 & 6.0 & 1.8 & 6.0 & 0.1 & l \\
\hline Acenaphthene & 0.1 & 7.1 & 0.9 & 3.0 & l & l \\
\hline Fluorene & 0.2 & 5.6 & 3 & 10.0 & 0.1 & 8.3 \\
\hline Phenanthrene & 0.2 & 5.5 & 2.1 & 7.0 & 0.1 & 1.1 \\
\hline Anthracene & 0.3 & 7.6 & 2.1 & 7.0 & 0.2 & 2.1 \\
\hline Pyrene & 0.2 & 6.4 & 3.6 & 12.0 & 0.2 & l \\
\hline Fluoroanthene & 0.2 & 6.2 & 2.1 & 7.0 & 0.2 & l \\
\hline 1,2-Benzanthracene & 0.1 & 6.2 & 2.1 & 7.0 & 0.2 & 6 \\
\hline Chrysene & 0.1 & 11.0 & 1.5 & 5.0 & 0.2 & 10.6 \\
\hline Benzo(b)fluoroanthene & 0.2 & 10.5 & 2.7 & 9.0 & 0.1 & / \\
\hline Benzo(k)fluoroanthene & 0.2 & 8.6 & 1.8 & 6.0 & 0.1 & l \\
\hline Benzo[a]pyrene & 0.3 & 7.2 & 3.6 & 12.0 & 0.1 & / \\
\hline Indeno(1,2,3 cd)pyrene & 0.8 & 9.2 & 3.6 & 12.0 & 0.3 & l \\
\hline Dibenz(ah)anthracene & 0.6 & 11.3 & l & / & 0.3 & l \\
\hline Benzo(ghi)perylene & 0.8 & 11.9 & 1.8 & 6.0 & 0.3 & l \\
\hline
\end{tabular}

MDL values calculated with a $99 \%$ confidence interval

/ not determined 
Table 5 Results for roofing felt extractions with ultrapure water, measured with a PAL SPME

Arrow $(250 \mu \mathrm{m} \times 20 \mathrm{~mm}$, $10.2 \mu \mathrm{L})$ : leached concentrations (estimated from calibrations for naphthalene and acenaphthylene for all other compounds) and relative standard deviations (RSD) at calculated concentrations

\begin{tabular}{llll}
\hline Compound & CAS Nr. & $\begin{array}{l}\text { Concentration leached } \\
\text { into water per gram } \\
\text { (ng L })^{-1}\end{array}$ & $\begin{array}{l}\text { RSD } \\
\text { (\%) }\end{array}$ \\
\hline Naphthalene & & 15 & 5.2 \\
2-Vinylnaphthalene & $91-20-30$ & 27 & 4.4 \\
Biphenylene & $827-54-3$ & 14 & 9.4 \\
[2-(Naphth-2-yl)vinyl]-methyl sulfone & $259-79-0$ & 4.9 \\
1-Isoquinolinecarbonitrile & Not available & 13 & 5.2 \\
5-Isoquinolinecarbonitrile & $1198-30-70$ & 24 & 4.6 \\
Benz(a)azulene & $27655-41-0$ & 13 & 5.1 \\
Acenaphthylene & $246-02-6$ & 16 & 5.6 \\
2,3-Naphthalenedicarbonitrile & $208-96-8$ & 38 & 4.0 \\
Diazene, 1-methoxy-2-[2-(1-naphthyl)ethenyl]-2-oxide- & Not available & 14 & 3.3 \\
\hline
\end{tabular}

inside the vials act as a second organic, hydrophobic phase. Since the sorptive properties of PAHs increase with their molecular weight, larger compounds are difficult to remove from this phase without a solvent extraction step. In addition to the two abovementioned PAHs, further compounds have been tentatively identified via their mass spectral information in the NIST library. These compounds and their estimated concentrations (converted from $300 \mathrm{mg}$ to $1 \mathrm{~g}$ ) are summarized in Table 5.

Latter concentrations can be expected to be leached into $1 \mathrm{~L}$ of water, which is exposed to $1 \mathrm{~g}$ of roofing felt under the extraction conditions given above. Since the used calibration standards contained the 16 EPA PAHs, these results were estimated using the calibration functions of naphthalene (for naphthalene and 2-vinylnaphthalene) and acenaphthylene (for all other compounds). It should however be noted that only PAHs and structurally similar substances such as heterocycles or substituted PAHs were taken into account during these measurements.

Despite the minor concentrations recovered in this smallscale experiment, the large quantities of, e.g., bitumen-based waterproofing materials that are applied globally could still account for a significant contribution to the overall anthropogenic discharge of PAHs into the environment. Further assessment of these contributions should involve influences by temperature, acidity, and UV radiation.

\section{Conclusions}

With PAL SPME Arrow, it was possible to measure PAHs from the freely dissolved fraction in aqueous samples down to the low nanogram-per-liter range or even below. For many compounds, this also applied if they had to be extracted from filtered groundwater. Achieved extraction yields and resulting sensitivities clearly benefit from the enlarged sorption phases of PAL SPME Arrow while all advantages of the classical SPME fiber are maintained.

As demonstrated in correlation with SBSE literature, the beneficial effect of increased sorption phase volumes declines with further increasing phase volumes, since the phase ratio between sample and sorption phase becomes less optimal unless significantly larger sample volumes in the range of liters are used. Since the handling of latter sample dimensions as well as the SBSE technique itself is more difficult to automate, PAL SPME Arrow might be a more effective solution in terms of combining maximal extraction efficiency with a fully automatable extraction device and sample size.

The only drawback of this new option in terms of the mandatory, slight widening of the injector port is considered less critical when compared to the additional thermal desorption equipment that is required for SBSE bars.

In addition, the increased mechanical robustness of PAL SPME Arrow facilitates extended, unattended measurement series typically found in routine laboratories.

Lastly, the enlarged sorption phase dimensions and the design principle of PAL SPME Arrow can be advantageous for the realization of new sorption phase materials and combinations. For instance, the enlarged surface area might enhance the effects of carbon nanomaterials, which exhibit promising potential as upcoming sorption phase materials [28, 29].

Acknowledgments We gratefully acknowledge the support by CTC Analytics AG and BGB Analytik AG of Switzerland during the integration of PAL SPME Arrow into the PAL RTC autosampler. Especially, Beat Schilling of BGB Analytik AG has been of invaluable assistance for the idea of PAL SPME Arrow becoming a reality. We would also like to thank Shimadzu Europa GmbH for the support concerning the GCMS instrumentation.

\section{Compliance with ethical standards}

Conflict of interest In spite of the support mentioned under acknowledgements, no conflict of interest influenced the work 
presented herein. The findings and conclusions in this report are those of the authors and do not represent the official views or positions of the supporting companies.

Open Access This article is distributed under the terms of the Creative Commons Attribution 4.0 International License (http:// creativecommons.org/licenses/by/4.0/), which permits unrestricted use, distribution, and reproduction in any medium, provided you give appropriate credit to the original author(s) and the source, provide a link to the Creative Commons license, and indicate if changes were made.

\section{References}

1. Belardi RP, Pawliszyn JB (1989) The application of chemically modified fused silica fibers in the extraction of organics from water matrix samples and their rapid transfer to capillary columns. Water Pollut Res J Can 24:179-191

2. Spietelun A, Kloskowski A, Chrzanowski W, Namieśnik J (2012) Understanding solid-phase microextraction: key factors influencing the extraction process and trends in improving the technique. Chem Rev (Washington, DC, U S) 113(3):1667-1685

3. Laaks J, Jochmann MA, Schmidt TC (2012) Solvent-free microextraction techniques in gas chromatography. Anal Bioanal Chem 402(2):565-571

4. García-Falcón MS, Cancho-Grande B, Simal-Gándara J (2004) Stirring bar sorptive extraction in the determination of PAHs in drinking waters. Water Res 38(7):1679-1684

5. Huang S, He S, Xu H, Wu P, Jiang R, Zhu F, Luan T, Ouyang G (2015) Monitoring of persistent organic pollutants in seawater of the Pearl River Estuary with rapid on-site active SPME sampling technique. Environ Pollut 200:149-158

6. Kole PL, Venkatesh G, Kotecha J, Sheshala R (2011) Recent advances in sample preparation techniques for effective bioanalytical methods. Biomed Chromatogr 25(1-2):199-217

7. Bagheri H, Piri-Moghadam H, Naderi M (2012) Towards greater mechanical, thermal and chemical stability in solid-phase microextraction. TrAC Trends Anal Chem 34:126-139

8. Baltussen E, Sandra P, David F, Cramers C (1999) Stir bar sorptive extraction (SBSE), a novel extraction technique for aqueous samples: theory and principles. J Microcolumn Sep 11(10):737-747

9. Es'haghi Z, Taghizade S, Mazloomi-Bajestani A (2014) Arsenic removal from water/wastewater using nanoparticle-assisted hollow fiber solid-phase microextraction combined with hydride generation-atomic fluorescence spectroscopy. J Iran Chem Soc 11(5):1421-1428

10. Kokosa JM, Przyjazny AJ, Jeannot MA (2009) Solvent microextraction: theory and practice. Wiley, Hoboken, N.J.

11. Tienpont B, David F, Bicchi C, Sandra P (2000) High capacity headspace sorptive extraction. J Microcolumn Sep 12(11):577-584

12. Purcaro G, Moret S, Conte LS (2013) Overview on polycyclic aromatic hydrocarbons: occurrence, legislation and innovative determination in foods. Talanta 105:292-305

13. Rasoulzadeh Y, Mortazavi SB, Yousefi AA, Khavanin A (2011) Decreasing polycyclic aromatic hydrocarbons emission from bitumen using alternative bitumen production process. J Hazard Mater 185(2-3):1156-1161

14. Horváth A, Clement A (2013) What can rainwater dissolve from bitumen shingle roof materials? Int J Eng Res Appl 3(6):194-199

15. Cheng X, Forsythe J, Peterkin E (2013) Some factors affecting SPME analysis and PAHs in Philadelphia's urban waterways. Water Res 47:2331-2340
16. Tsimeli K, Triantis T, Dimotikali D, Hiskia A (2008) Development of a rapid and sensitive method for the simultaneous determination of 1,2-dibromoethane, 1,4-dichlorobenzene and naphthalene residues in honey using HS-SPME coupled with GC-MS. Anal Chim Acta 617(1-2):64-71

17. Mader BT, Pankow JF (2000) Gas/solid partitioning of semivolatile organic compounds (SOCs) to air filters. 1. Partitioning of polychlorinated dibenzodioxins, polychlorinated dibenzofurans and polycyclic aromatic hydrocarbons to teflon membrane filters. Atmos Environ 34(28):4879-4887

18. Staudinger J, Roberts P (2001) A critical compilation of Henry's law constant temperature dependence relations for organic compounds in dilute aqueous solutions. Chemosphere 44(4):561-576

19. Qian Y, Posch T, Schmidt TC (2011) Sorption of polycyclic aromatic hydrocarbons (PAHs) on glass surfaces. Chemosphere 82(6): 859-865

20. Barco-Bonilla N, Romero-González R, Plaza-Bolaños P, Fernández-Moreno JL, Garrido Frenich A, Martínez Vidal JL (2011) Comprehensive analysis of polycyclic aromatic hydrocarbons in wastewater using stir bar sorptive extraction and gas chromatography coupled to tandem mass spectrometry. Anal Chim Acta 693(1-2):62-71

21. Louch D, Motlagh S, Pawliszyn JB (1992) Dynamics of organic compound extraction from water using liquid-coated fused silica fibers. Anal Chem 64:1187-1199

22. Pérez-Carrera E, León VML, Parra AG, González-Mazo E (2007) Simultaneous determination of pesticides, polycyclic aromatic hydrocarbons and polychlorinated biphenyls in seawater and interstitial marine water samples, using stir bar sorptive extraction-thermal desorption-gas chromatography-mass spectrometry. J Chromatogr A 1170(1-2):82-90

23. Sprunger L, Proctor A, Acree WL, Abraham MH (2007) Characterization of the sorption of gaseous and organic solutes onto polydimethyl siloxane solid-phase microextraction surfaces using the Abraham model. J Chromatogr A 1175: 162-173

24. Bradley J-C, Abraham MH, Acree WE, Lang ASID, Beck SN, Bulger DA, Clark EA, Condron LN, Costa ST, Curtin EM, Kurtu SB, Mangir MI, McBride MJ (2015) Determination of Abraham model solute descriptors for the monomeric and dimeric forms of trans-cinnamic acid using measured solubilities from the Open Notebook Science Challenge. Chem Cent J 9:11

25. Zimmermann T, Ensinger WJ, Schmidt TC (2006) Depletion solid-phase microextraction for the evaluation of fiber-sample partition coefficients of pesticides. J Chromatogr A 1102(1-2): $51-59$

26. Keith LH, Crummett W, Deegan J, Libby RA, Taylor JK, Wentler G (1983) Principles of environmental analysis. Anal Chem 55(14): 2210-2218

27. King AJ, Readman JW, Zhou JL (2004) Determination of polycyclic aromatic hydrocarbons in water by solid-phase microextraction-gas chromatography-mass spectrometry. Anal Chim Acta 523(2):259-267

28. Huffer T, Osorio X, Jochmann M, Schilling B, Schmidt T (2013) Multi-walled carbon nanotubes as sorptive material for solventless in-tube microextraction (ITEX2) - a factorial design study. Anal Bioanal Chem 405(26):8387-8395

29. Tang P-P, Cai J-B, Su Q-D (2009) Carbon nanotubes coated fiber for solid-phase microextraction of bovine fibrinogen and bovine serum albumin. J Chin Chem Soc (Taipei, Taiwan) 56(6):1128-1138 